\title{
Isolierung von Choleinsäure, Stearinsäure und Cholesterin aus Rindergallensteinen. \\ Von
}

Hans Fischer und P. Meyer.

(Aus der II. med. Klinik zu München.)

(Der Redaktion zugegangen am 26. Oktober 1911).

Zur Bilirubingewinnung aus Rindergallensteinen wurde das trockene Pulver der Gallensteine zunächst erschöpfend mit Äther ausgezogen. Beim langsamen Eindunsten des Extraktes schied sich eine reichliche Menge von Krystallen ab, die nach mehrmaligem Umkrystallisieren aus verdünntem Alkohol den konstanten Schmelzpunkt $185-188^{\circ}$ zeigten. Die Elementaranalyse der bei $100^{\circ}$ über $\mathrm{P}_{2} \mathrm{O}_{5}$ zur Konstanz getrockneten Substanz ergab:

0,1953 g Substanz gaben $0,5243 \mathrm{~g} \mathrm{CO}_{2}$ und $0,1815 \mathrm{~g}$ Wasser.
Berechnet für $\mathrm{C}_{24} \mathrm{H}_{40} \mathrm{O}_{4}$ :
Gefunden :
C. 73,46
73,22
H 10,21
10,40

Die Titration der in absolutem Alkohol gelösten Substanz mit Phenolphthalein als Indikator ergab:

$0,1691 \mathrm{~g}$ Substanz verbrauchten $4,4 \mathrm{ccm}$ 1/10-n-Natronlauge. Hieraus berechnet sich Molekulargewicht $=384$, während Choleinsäure 392 verlangt.

Optische Bestimmung:

0,0983 g Substanz in absolutem Alkohol gelöst. Gesamtgewicht 3,2519. $d_{20}=0,81$. Drehung im $1 \mathrm{dm}-R o h r 1,18^{\circ}$ nach rechts. Mithin

$$
[\alpha]_{\mathrm{d}}=+48,20^{\circ} \text {. }
$$


Ein zweites Präparat, das aus einer anderen Partie Rindergallensteine nach dem gleichen Verfahren gewonnen war, lieferte die gleichen Resultate.

Analyse: $0,1485 \mathrm{~g}$ Substanz gaben $0,3986 \mathrm{~g} \mathrm{CO}_{2}$ und $0,1422 \mathrm{~g}$ Wasser.

Berechnet:

C. 73,46

H 10,21
Gefunden :

73,21

10,71

Optische Bestimmung:

$0,0378 \mathrm{~g}$ Substanz in absolutem Alkohol gelöst. Gesamtgewicht der Lösung 1,2336. $d_{20}=0,81$. Drehung im $1 \mathrm{dm}-$ Rohr $1,21^{\circ}$ nach rechts. Mithin

$$
[\alpha]_{d}=+48,72^{\circ} \text {. }
$$

Die Fluorescenzprobe mit Schwefelsäure war intensiv positiv, ebenso die Pettenkofersche Probe, während die Myliussche Jodreaktion absolut negativ war.

Nach Schmelzpunkt, Analyse, Titration und optischer Bestimmung (Langheld') fand für Choleinsäure 47,97, Pregl²) 48,47) kann es keinem Zweifel unterliegen, daß hier Choleinsäure vorliegt. Eine Eigenschaft stimmt jedoch nicht mit den Angaben über Choleinsäure, und das ist der Geschmack. Unsere Säure ist absolut geschmacklos, während Hammarsten ${ }^{3}$ ) den Geschmack als intensiv bitter angibt.

Leider haben wir weitere Angaben über den Geschmack der Gallensäuren nicht gefunden, nach Hammarsten (l. c.) schmecken alle drei spezifischen Gallensäuren intensiv bitter. Nach unseren Untersuchungen schmeckt Cholalsäure intensiv bitter, Desoxycholsäure (aus Stuhl) ganz schwach bitter, Choleinsäure und Lihocholsäure sind absolut geschmacklos.

Mit obigen Angaben sollen indessen die Befunde Hammarstens durchaus nicht bezweifelt werden, sondern wir wollen nur die Aufmerksamkeit der auf dem Gebiet arbeitenden

1) Langheld, Ber. 41, S. 384.

2) Pregl, Diese Zeitschrift, Bd. 65, S. 158.

$\left.{ }^{8}\right)$ Hammarsten in $\mathrm{Abderhaldens}$ biochemischen Arbeitsmethoden, S. 661. 
Forscher auf diesen Punkt lenken, weil es hierdurch unter Umständen möglich ist, zwischen den einzelnen Gallensäuren weitere Differenzen zu finden, die analytisch vielleicht gar nicht nachweisbar sind.

Obwohl sich Choleinsäure und Desoxycholsäure durch Elementaranalyse, Leichtlöslichkeit des Ammonium- und Natronsalzes und spezifische Drehung von der Lihocholsäure ${ }^{1}$ ) unterscheiden, haben wir noch den Mischschmelzpunkt der Lihocholsäure mit Choleinsäure und Desoxycholsäure genommen. Es ergab sich in beiden Fällen eine erhebliche Depression und unscharfer Schmelzpunkt. (Mit Choleinsäure $160^{\circ}$ unscharf, mit Desoxycholsäure 165-171.)

Die obenerwähnte Mutterlauge der Choleinsäure wurde, nachdem sie keine weiteren Krystalle mehr abschied, mit Äther verdünnt und mit Ammoniak ausgeschüttelt.

In der Ätherlösung bleibt neben Schmiere Cholesterin, das wir durch Schmelzpunkt, Reaktionen und Überführung in das von Windaus beschriebene Cholesterindibromid ${ }^{2}$ ) identifizierten.

Das ammoniakalische Extrakt wurde nach starker Verdünnung mit $\mathrm{H}_{2} \mathrm{O}$ durch Chlorbaryum gefällt, der voluminöse Niederschlag abgesaugt, mit Wasser gewaschen und mit $96 \%$ igem Alkohol erschöpfend ausgekocht.

Der Rückstand wurde mit Salzsäure angerieben, abgesaugt und hierauf aus verdünntem Alkohol umkrystallisiert, bis der konstante Schmelzpunkt 68-690 erreicht war. Der Körper charakterisierte sich in all seinen Eigenschaften als eine Fettsäure und die Elementaranalyse bestätigte, daß Stearinsäure vorlag. Wasser.

$0,1470 \mathrm{~g}$ Substanz gaben $0,4088 \mathrm{~g} \mathrm{CO}_{2}$ und $0,1685 \mathrm{~g}$

Berechnet für $\mathrm{C}_{18} \mathrm{H}_{36} \mathrm{O}_{2}$ :

C 75,97

Gefunden:

$\mathrm{H} 12,76$

75,84

12,83

Die Ausbeuten an allen drei isolierten Körpern waren sehr gering, insbesondere ist Cholesterin nur in Spuren in den

1) H. Fischer, I. Mitteilung über Gallenfarbstoffe.

2) Windaus, Chem. Zeitung, 30.1011.

Hoppe-Seyler's Zeitschrift f. physiol. Chemie. LXXVI. 
Rindergallensteinen vorhanden (Cholesterin ca. 0,1\%, Stearinsäure $1 \%$, Choleinsäure $0,5 \%$ ).

Natürlich gelten die angegebenen Zahlen nur ganz approximativ, da die Verarbeitung keineswegs eine quantitative war. Auffallend ist immerhin, daß alle drei Körper in freiem Zustand vorkommen. Dies spricht für die schon von vielen Autoren vertretene Ansicht, daß bei der Cholelithiasis der Rinder Fäulnisprozesse eine hervorragende Rolle spielen.

Bei der menschlichen Cholelithiasis dagegen scheinen andere Prozesse die Ursache zu sein; wir werden hierauf später eingehen. 